\title{
Integration of rotational angiography enables better dose planning in Gamma Knife radiosurgery for brain arteriovenous malformations
}

\author{
Hirotaka Hasegawa, MD, ${ }^{1}$ Shunya Hanakita, MD, PhD, ${ }^{1}$ Masahiro Shin, MD, PhD, ${ }^{1}$ \\ Mariko Kawashima, MD, ${ }^{1}$ Taichi Kin, MD, PhD, ${ }^{1}$ Wataru Takahashi, MD, PhD, ${ }^{2}$ Yuichi Suzuki, PhD, ${ }^{2}$ \\ Yuki Shinya, MD, ${ }^{1}$ Hideaki Ono, MD, PhD, ${ }^{1}$ Masaaki Shojima, MD, PhD, ${ }^{1}$ \\ Hirofumi Nakatomi, MD, PhD, ${ }^{1}$ and Nobuhito Saito, MD, PhD'
}

Departments of ${ }^{1}$ Neurosurgery and ${ }^{2}$ Radiology, University of Tokyo Hospital, Tokyo, Japan

OBJECTIVE In Gamma Knife radiosurgery (GKS) for arteriovenous malformations (AVMs), CT angiography (CTA), MRI, and digital subtraction angiography (DSA) are generally used to define the nidus. Although the AVM angioarchitecture can be visualized with superior resolution using rotational angiography (RA), the efficacy of integrating RA into the GKS treatment planning process has not been elucidated.

METHODS Using data collected from 25 consecutive patients with AVMs who were treated with GKS at the authors' institution, two neurosurgeons independently created treatment plans for each patient before and after RA integration. For all patients, MR angiography, contrasted T1 imaging, CTA, DSA, and RA were performed before treatment. The prescription isodose volume before $\left(\mathrm{PIV}_{\mathrm{B}}\right)$ and after $\left(\mathrm{PIV}_{\mathrm{A}}\right)$ RA integration was measured. For reference purposes, a reference target volume (RTV) for each nidus was determined by two other physicians independent of the planning surgeons, and the RTV covered by the PIV (RTV $\mathrm{PIV}_{\text {) }}$ ) was established. The undertreated volume ratio (UVR), overtreated volume ratio (OVR), and Paddick's conformal index $(\mathrm{Cl})$, which were calculated as $\mathrm{RTV}_{\mathrm{PIV}} / \mathrm{RTV}, \mathrm{RTV}_{\mathrm{PIV}} / \mathrm{PIV}$, and $\left(\mathrm{RTV}_{\mathrm{PIV}}\right)^{2 /}$ $(\mathrm{RTV} \times \mathrm{PIV})$, respectively, were measured by each neurosurgeon before and after RA integration, and the surgeons' values at each point were averaged. Wilcoxon signed-rank tests were used to compare the values obtained before and after RA integration. The percentage change from before to after RA integration was calculated for the average UVR (\% $\%$ UVRave), OVR ( $\% \Delta O V R a v e)$, and $\mathrm{Cl}(\% \Delta$ Clave) in each patient, as ([value after RA integration]/[value before RA integration] -1$) \times 100$. The relationships between prior histories and these percentage change values were examined using Wilcoxon signed-rank tests.

RESULTS The average values obtained by the two surgeons for the median UVR, OVR, and $\mathrm{Cl}$ were $0.854,0.445$, and 0.367 before RA integration and $0.882,0.478$, and 0.463 after RA integration, respectively. All variables significantly improved after compared with before RA integration (UVR, $p=0.009 ;$ OVR, $p<0.001 ; \mathrm{Cl}, p<0.001$ ). Prior hemorrhage was significantly associated with larger $\% \Delta \mathrm{OVR}_{\text {ave }}$ (median $20.8 \%$ vs $7.2 \% ; p=0.023$ ) and $\% \Delta \mathrm{Cl}_{\text {ave }}$ (median $33.9 \%$ vs $13.8 \% ; p=0.014$ ), but not $\% \Delta U V_{\text {ave }}$ (median $4.7 \%$ vs $4.0 \% ; p=0.449$ ).

CONCLUSIONS Integrating RA into GKS treatment planning may permit better dose planning owing to clearer visualization of the nidus and, as such, may reduce undertreatment and waste irradiation. Further studies examining whether the observed RA-related improvement in dose planning also improves the radiosurgical outcome are needed.

https://thejns.org/doi/abs/10.3171/2018.7.GKS181565

KEYWORDS arteriovenous malformation; dose planning; Gamma Knife radiosurgery; rotational angiography; vascular disorders; stereotactic radiosurgery

\footnotetext{
ABBREVIATIONS AVM = arteriovenous malformation; $\mathrm{Cl}=$ conformity index; $\mathrm{CTA}=\mathrm{CT}$ angiography; $\mathrm{DSA}=$ digital subtraction angiography; FOV = field of view; $\mathrm{GKS}=$ Gamma Knife radiosurgery; HiRes-XperCT = high-resolution XperCT; OVR = overtreated volume ratio; PIV = prescription isodose volume; RA = rotational angiography; RTV = reference target volume; TOF-MRA = time-of-flight MR angiography; UVR = undertreated volume ratio; 3DRA = 3D rotational angiography.

SUBMITTED May 31,2018. ACCEPTED July 16, 2018. INCLUDE WHEN CITING DOI: 10.3171/2018.7.GKS181565.
} 
$\mathrm{G}$ AMMA Knife radiosurgery (GKS) provides a minimally invasive treatment option for small- to medium-size brain arteriovenous malformations (AVMs) and has a 65\%-85\% obliteration rate after a 2to 3-year latency period.7,13,14,19,20,34 During GKS planning, surgeons circumscribe the nidus by meticulously examining radiological images. Initially, only biplanar digital subtraction angiography (DSA) was used; however, owing to modern advances in radiological techniques and the development of planning software, CT angiography (CTA) and MRI have become valuable tools, as they enable axial image-based radiosurgical planning, which improves the quality of target contouring., 47,20,21,24 Since radiotherapy planning is solely dependent on radiological images, using high-resolution images is essential, as such images can theoretically reduce radiation waste and avoid suboptimal coverage of the targets, ultimately improving the radiosurgical outcome. ${ }^{12,22}$ However, the imaging modality best suited to provide high-resolution images for GKS planning remains unknown.

Rotational angiography (RA), performed with a coneshaped X-ray beam and newer-generation flat-panel detectors on a modern $\mathrm{C}$-arm system, can provide excellent spatial resolution of the angioarchitecture of vascular lesions. ${ }^{27,28,37}$ Although it is likely that the integration of RA would improve GKS dose planning, few studies examining the efficacy of integrating RA into the GKS treatment planning process have been performed., ${ }^{6,30}$ At our institution, we have routinely used RA for GKS planning since 2015. Therefore, the aim of the present study was to examine the efficacy of integrating RA into the dose planning process for AVM treatment.

\section{Methods \\ Patients}

We collected data from 29 consecutive patients with AVM who were treated with GKS (Gamma Knife 4C; Elekta AB) in our hospital between September 2015 and September 2016. After excluding two patients with large AVMs ( $\geq 35 \mathrm{~mm}$ in maximum diameter) and two patients with incomplete data, 25 patients with 25 AVMs were enrolled. This study was approved by the institutional review board of our hospital, and all patients provided written informed consent to participate.

\section{Protocols for CTA, MRI, and Angiographic Image Acquisition}

We routinely performed time-of-flight MR angiography (TOF-MRA), contrast-enhanced T1-weighted imaging, and CTA the day before GKS. For CTA, the matrix size was $512 \times 512$ pixels with a $200 \times 200 \mathrm{~mm}^{2}$ field of view $(\mathrm{FOV})$, yielding a spatial resolution of $0.39 \times 0.39$ $\mathrm{mm}^{2}$ with a $0.5-\mathrm{mm}$ slice thickness and $0.3-\mathrm{mm}$ slice overlap (Acquilion One; Canon Medical Systems).

We acquired MR images with a 3.0-T MRI scanner (MAGNETOM Skyra, Siemens Healthineers; or Signa HDxt, GE Healthcare). When using the MAGNETOM Skyra, the parameters for TOF-MRA were as follows: matrix size $448 \times 269$ pixels; FOV $200 \times 200 \mathrm{~mm}^{2}$; spatial resolution $0.45 \times 0.74 \mathrm{~mm}^{2}$; and slice thickness 0.8 $\mathrm{mm}$. The parameters for acquiring contrast-enhanced T1weighted images on the MAGNETOM Skyra were as follows: matrix size $256 \times 256$ pixels; FOV $160 \times 160 \mathrm{~mm}^{2}$; spatial resolution $0.63 \times 0.63 \mathrm{~mm}^{2}$; and slice thickness $0.8 \mathrm{~mm}$. When using the Signa HDxt, the parameters for TOF-MRA were as follows: matrix size $512 \times 256$ pixels; FOV $200 \times 200 \mathrm{~mm}^{2}$; spatial resolution $0.39 \times 0.78 \mathrm{~mm}^{2}$; and slice thickness $0.8 \mathrm{~mm}$. The parameters for acquiring contrast-enhanced T1-weighted images on the Signa HDxt were as follows: matrix size $256 \times 224$ pixels; FOV 160 $\times 160 \mathrm{~mm}^{2}$; spatial resolution $0.63 \times 0.71 \mathrm{~mm}^{2}$; and slice thickness $0.8 \mathrm{~mm}$.

Angiography was performed after head fixation using the Leksell G frame (Elekta AB) on the day of GKS using the Allura Xper FD20/10 x-ray system (Philips Healthcare). Along with conventional DSA, RA was performed using two different preprogrammed modes, namely the $3 \mathrm{D}$ rotational angiography (3DRA; Philips Healthcare) or high-resolution XperCT (HiRes-XperCT; Philips Healthcare) modes. In both modes, continuous image acquisition was performed during a single rotation of the C-arm. The 3DRA mode used a quick rotation $(4 \mathrm{sec})$ with a variablesized (8- to 19-inch) detector, whereas the HiRes-XperCT mode used a slow, long acquisition (20 sec) with an 8-inch detector (Table 1). Although the HiRes-XperCT mode provided superior spatial resolution, the 3DRA mode with a relatively large detector size (10.5- to 13-inch) was generally preferred for better image coregistration, whereas HiRes-XperCT was preferred for small faint niduses. The obtained volume data set was transferred to the workstation (XtraVision; Philips Healthcare) and postprocessing was performed to create CT-like images with a $256 \times$ $256 \times 256$ or $512 \times 512 \times 512$ resolution voxel matrix in a changeable FOV, yielding a spatial resolution of $0.15-0.4$ $\mathrm{mm}$, depending on the size of the detector, voxel matrix, and FOV. The injection rate of the contrast medium was determined individually by the neuroendovascular surgeons (M.S. and H.O.) based on the findings of DSA, usually $3.0-4.0 \mathrm{ml} / \mathrm{sec}$ (3DRA) or $1.0-1.5 \mathrm{ml} / \mathrm{sec}$ (HiResXperCT). The start time of the acquisition was usually set as the time when a nidus was fully filled with contrast

TABLE 1. Acquisition parameters of 3DRA and HiRes-XperCT

\begin{tabular}{|c|c|c|}
\hline Parameter & 3DRA & HiRes-XperCT \\
\hline Scan time & $4 \mathrm{sec}$ & $20 \mathrm{sec}$ \\
\hline Frame rate & 30 frames/sec & 30 frames $/ \mathrm{sec}$ \\
\hline Rotation angle & $240^{\circ}$ & $240^{\circ}$ \\
\hline C-arm speed & $55^{\circ} / \mathrm{sec}$ & $10 \% \mathrm{sec}$ \\
\hline Detector size & Variable & Small (8 in.) \\
\hline Focus size & Small & Small \\
\hline $\begin{array}{l}\text { Acquisition } \\
\text { matrix }\end{array}$ & $1024 \times 1024$ & $1024 \times 1024$ \\
\hline $\begin{array}{l}\text { Pixel size (on } \\
\text { detector) }\end{array}$ & $\begin{array}{l}\text { Variable, binned or } \\
\text { unbinned }\end{array}$ & $154 \mu \mathrm{m}$, unbinned \\
\hline Tube voltage & Variable (50-125 kV) & Low (80 kV) \\
\hline Main target & Vascular anatomy & $\begin{array}{l}\text { High-contrast objects including } \\
\text { vascular \& bony anatomy }\end{array}$ \\
\hline
\end{tabular}


medium just before the appearance of drainers, leading to a preacquisition delay of approximately $3 \mathrm{sec}$. The acquisition times were $4 \mathrm{sec}$ (3DRA) and $20 \mathrm{sec}$ (HiRes-Xper$\mathrm{CT})$. Thus, the total amounts of contrast medium required for a single acquisition were roughly 20-30 $\mathrm{ml}$ (3DRA) and 25-40 $\mathrm{ml}$ (HiRes-XperCT). Each of the acquisition procedures was performed for $<2 \mathrm{~min}$. The acquired data were subsequently transferred to the treatment software (GammaPlan version 10.1.1; Elekta AB). Data merging among the imaging modalities was conducted automatically using a preprogrammed function in the GammaPlan software. Stereotactic CT with a $0.50-\mathrm{mm}$ slice thickness was used for stereotactic imaging.

\section{Radiosurgical Planning}

Two neurosurgeons (H.H. and M.K.) retrospectively created two treatment plans (one before and one after RA integration) for each patient. Aiming for a standardized planning method to reduce inter- and intraobserver variabilities, ${ }^{1,39,41}$ we used the following protocols during dose planning; 1) the radiosurgical target should be determined by meticulously comparing images acquired using a variety of imaging studies; 2) each shot should be placed inside the radiosurgical target; 3) if possible, feeders and drainers should be excluded from the radiosurgical target; 4) if it is difficult to judge whether a vessel belongs to a nidus, feeder, or drainer, trace the vessel both downstream and upstream and determine where it belongs; 5) radiosurgical doses should be prescribed with a $50 \% \pm 5 \%$ isodose line; and 6) the gamma angle should be fixed at $90^{\circ}$.

\section{Statistical Analysis}

By using a preinstalled function within GammaPlan, the contours of the true niduses were independently circumscribed for reference purposes with polygonal lines on each slice, and we meticulously compared all of the available tomographic images (TOF-MRA, contrast-enhanced T1-weighted, CTA, and RA images, among others). Brain tissues interposing inside a nidus and extranidal feeders and drainers were carefully segregated and excluded from the true niduses. Using the dose-volume calculation function within GammaPlan, a reference target volume (RTV) for each nidus was determined. Since determining the AVM microanatomies was a subjective judgement, two physicians (Y.S., a neurosurgeon, and W.T., a radiologist) worked together to create the RTV to ensure objectivity.

The prescription isodose volume before $\left(\mathrm{PIV}_{\mathrm{B}}\right)$ and after $\left(\mathrm{PIV}_{\mathrm{A}}\right)$ the integration of RA was calculated. The RTV

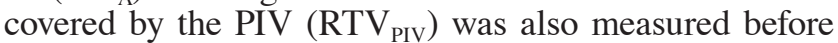
and after RA integration. For both treatment plans (before and after RA), each of the two surgeons calculated the undertreated volume ratio (UVR) and overtreated volume ratio (OVR) as $\mathrm{RTV}_{\mathrm{PIV}} / \mathrm{RTV}$ and $\mathrm{RTV}_{\mathrm{PIV}} / \mathrm{PIV}$, respectively; ${ }^{25}$ both of which would yield 1 if a nidus was perfectly contoured. Figure 1 shows a representative illustration of the PIV, RTV, and UTV and OTV. Additionally, each surgeon obtained the conformity index (CI) before and after RA integration, which was defined according to Paddick's criteria as follows: $\left(\mathrm{RTV}_{\mathrm{PIV}}\right)^{2} /(\mathrm{RTV} \times \mathrm{PIV}){ }^{25}$ First, each surgeon's values, as well as the average values from both surgeons, for each plan (before and after RA integration) were compared using Wilcoxon signed-rank tests. Second, to assess how prior events (hemorrhage, radiotherapy, and surgery/embolization) affected the efficacy of the present method, the percentage change from before to after RA integration was calculated for the average UVR $\left(\% \Delta \mathrm{UVR}_{\text {ave }}\right)$, OVR $\left(\% \Delta \mathrm{OVR}_{\text {ave }}\right)$, and $\mathrm{CI}\left(\% \Delta \mathrm{CI}_{\mathrm{ave}}\right)$ in each patient, as ([value after RA integration]/[value before RA integration] -1$) \times 100$. The relationships between the prior events and each percentage change were analyzed with Mann-Whitney U-tests. Differences were considered significant at $p<0.05$. All statistical analyses were performed using JMP Pro 13.0 (SAS Institute Inc.).

\section{Results}

The baseline characteristics of the patients are listed in
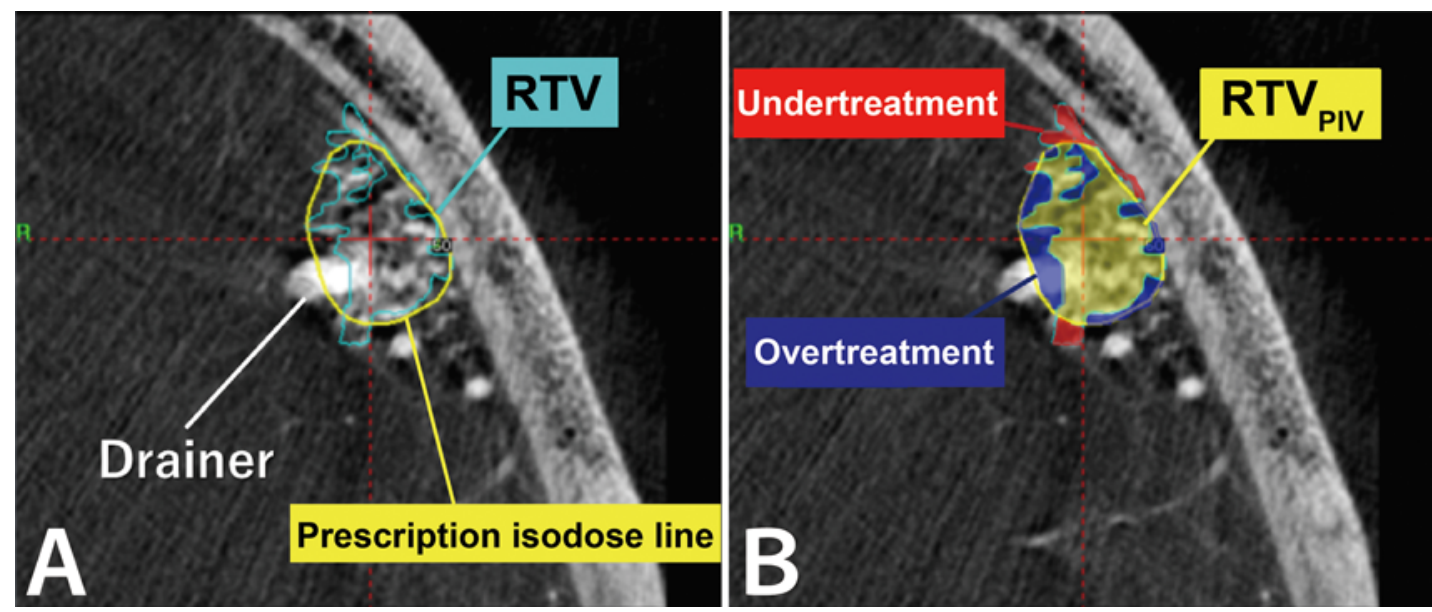

FIG. 1. Representative images showing the prescription isodose volume (PIV), reference target volume (RTV), RTV covered


indicates the RTV (A). Note that an extranidal drainer is excluded from the RTV. Using the same axial image as in A, the spatial

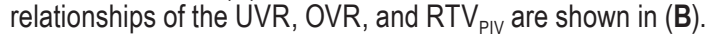


TABLE 2. Baseline characteristics of the patients

\begin{tabular}{lc}
\hline \multicolumn{1}{c}{ Variables } & Value (median) [\%] \\
\hline $\mathrm{N}$ & 25 \\
\hline Age at treatment, yrs & $15-67(43)$ \\
\hline Planned target volume, $\mathrm{ml}$ & $0.1-12.8(1.8)$ \\
\hline Maximum diameter, $\mathrm{mm}$ & $6-32(19)$ \\
\hline Male sex & $13[52]$ \\
\hline History of direct surgery & $2[8]$ \\
\hline History of embolization & $2[8]$ \\
\hline History of hemorrhage & $8[32]$ \\
\hline Redo GKS & $6[24]$ \\
\hline Location & $9[36]$ \\
\hline Frontal lobe & $8[32]$ \\
\hline Parietal lobe & $3[12]$ \\
\hline Temporal lobe & $2[8]$ \\
\hline Cerebellum & $3[12]$ \\
\hline BGL/thalamus & \\
\hline Spetzler-Martin grade & $7[28]$ \\
\hline I & $9[36]$ \\
\hline II & $9[36]$ \\
\hline III &
\end{tabular}

$\mathrm{BGL}=$ basal ganglia .

Table 2. Briefly, the median age, AVM volume, and maximal AVM diameter were 43 years (range 15-67 years), $1.8 \mathrm{ml}$ (range $0.1-12.8 \mathrm{~m}$ ), and $19 \mathrm{~mm}$ (range 6-32 $\mathrm{mm}$ ), respectively. Eight patients (32\%) had experienced hemorrhage before GKS, and 6 patients $(27 \%)$ had received GKS treatment previously. Two (8\%) patients had previously undergone direct surgery to the nidus, and two (8\%) had previously undergone endovascular embolization. In one patient, the nidus was very small, and thus a $70 \%$ isodose line was used.

The UVR, OVR, and CI values obtained by each surgeon for each treatment plan (before and after RA integration) are summarized in Table 3 . All values obtained by surgeon 1 (H.H.) significantly improved after RA integra- tion compared to before RA integration (UVR, $p=0.003$; OVR, $\mathrm{p}=0.001$; CI, $\mathrm{p}<0.001$ ). As for surgeon 2 (M.K.), although all of the values improved after RA integration, significant differences were only observed for the OVR ( $p$ $=0.040)$ and CI $(\mathrm{p}<0.001)$ values but not for the UVR (p $=0.129$ ) value. When the values obtained by each surgeon for each plan were averaged, all values were significantly improved after compared with before RA integration (UVR, $\mathrm{p}=0.009$; OVR, $\mathrm{p}<0.001 ; \mathrm{CI}, \mathrm{p}<0.001$ ).

The median values of $\% \Delta \mathrm{UVR}_{\text {ave }}, \% \Delta \mathrm{OVR}_{\text {ave }}$, and $\% \Delta \mathrm{CI}_{\text {ave }}$ were $+3.9 \%$ (range $-11.4 \%$ to $+51.6 \%$ ), $+12.6 \%$ (range $-4.5 \%$ to $+78.5 \%$ ), and $+18.6 \%$ (range $-3.9 \%$ to $+147.2 \%$ ), respectively. Prior hemorrhage was significantly associated with larger $\% \Delta O \mathrm{OVR}_{\text {ave }}$ (median $20.8 \%$ vs $7.2 \%$; $\mathrm{p}=0.023$ ) and $\% \Delta \mathrm{CI}_{\text {ave }}$ (median $33.9 \%$ vs $13.8 \% ; \mathrm{p}=$ 0.014 ) values but not $\% \Delta \mathrm{UVR}_{\text {ave }}$ values (median $4.7 \%$ vs $4.0 \% ; \mathrm{p}=0.449$ ), whereas a history of surgery/embolization or radiotherapy was not significantly associated with larger improvement in any of the average values.

Images from several illustrative cases are shown in Figs. 2-5.

\section{Discussion}

In the present study, we compared the dosimetric variables in GKS planning before and after the integration of RA and confirmed that the integration of RA reduced not only suboptimal coverage of the nidus but also the waste irradiation outside of the nidus, resulting in improved conformity. Conformity is one of the most important dosimetric factors for radiosurgery, ${ }^{29}$ with several authors suggesting that conformal radiotherapy significantly reduces radiation-induced adverse events by decreasing the toxicity to healthy tissues ${ }^{11,16,23}$ and that dose escalation delivered by conformal radiotherapy may improve the radiosurgical efficacy without increasing the toxicity. ${ }^{2,3}$ Although the efficacy of RA for other radiotherapy modalities (e.g., CyberKnife) has been studied previously, $, 10,18,36,38$ their results should not be applied unthoughtfully to GKS, as the radiosurgical planning process used in those modalities is totally different from that used in GKS. To date, few studies examining the efficacy of RA in GKS have been reported, and no detailed analyses of dosimetric variables like those

TABLE 3. UVR, OVR, and $\mathrm{Cl}$ for two surgeons before and after integration of 3DRA

\begin{tabular}{|c|c|c|c|c|c|c|c|c|c|}
\hline \multirow[b]{2}{*}{ Variable } & \multicolumn{3}{|c|}{ Surgeon 1} & \multicolumn{3}{|c|}{ Surgeon 2} & \multicolumn{3}{|c|}{ Average } \\
\hline & Median (range) & $\%$ Change* $^{*}$ & $p$ Value & Median (range) & $\%$ Change $^{*}$ & $p$ Value & Median (range) & $\%$ Change $^{*}$ & $\mathrm{p}$ Value \\
\hline \multicolumn{10}{|l|}{ UVR } \\
\hline Before & $0.882(0.573-0.975)$ & \multirow{2}{*}{$+5.0 \%$} & \multirow{2}{*}{$0.003 \dagger$} & $0.826(0.398-0.965)$ & \multirow{2}{*}{$+1.3 \%$} & \multirow{2}{*}{0.129} & $0.868(0.498-0.945)$ & \multirow{2}{*}{$+2.5 \%$} & \multirow{2}{*}{$0.009 \dagger$} \\
\hline After & $0.926(0.803-0.989)$ & & & $0.837(0.595-0.972)$ & & & $0.890(0.730-0.958)$ & & \\
\hline \multicolumn{10}{|l|}{ OVR } \\
\hline Before & $0.452(0.111-0.762)$ & \multirow{2}{*}{$+12.2 \%$} & \multirow{2}{*}{$0.001 \dagger$} & $0.438(0.064-0.833)$ & \multirow{2}{*}{$+3.2 \%$} & \multirow{2}{*}{$0.040 \dagger$} & $0.419(0.088-0.798)$ & \multirow{2}{*}{$+15.8 \%$} & \multirow{2}{*}{$<0.001 \dagger$} \\
\hline After & $0.507(0.209-0.780)$ & & & $0.452(0.071-0.934)$ & & & $0.485(0.156-0.857)$ & & \\
\hline \multicolumn{10}{|l|}{$\mathrm{Cl}$} \\
\hline Before & $0.372(0.095-0.656)$ & \multirow{2}{*}{$+30.6 \%$} & \multirow{2}{*}{$<0.001 \dagger$} & $0.362(0.054-0.601)$ & \multirow{2}{*}{$+21.3 \%$} & \multirow{2}{*}{$<0.001 \dagger$} & $0.392(0.075-0.622)$ & \multirow{2}{*}{$+21.7 \%$} & \multirow{2}{*}{$<0.001 \uparrow$} \\
\hline After & $0.486(0.207-0.700)$ & & & $0.439(0.141-0.689)$ & & & $0.477(0.184-0.694)$ & & \\
\hline
\end{tabular}

${ }^{*} \mathrm{~A} p$ value $<0.05$ was considered significant.

$\dagger$ Changes for each median value. 


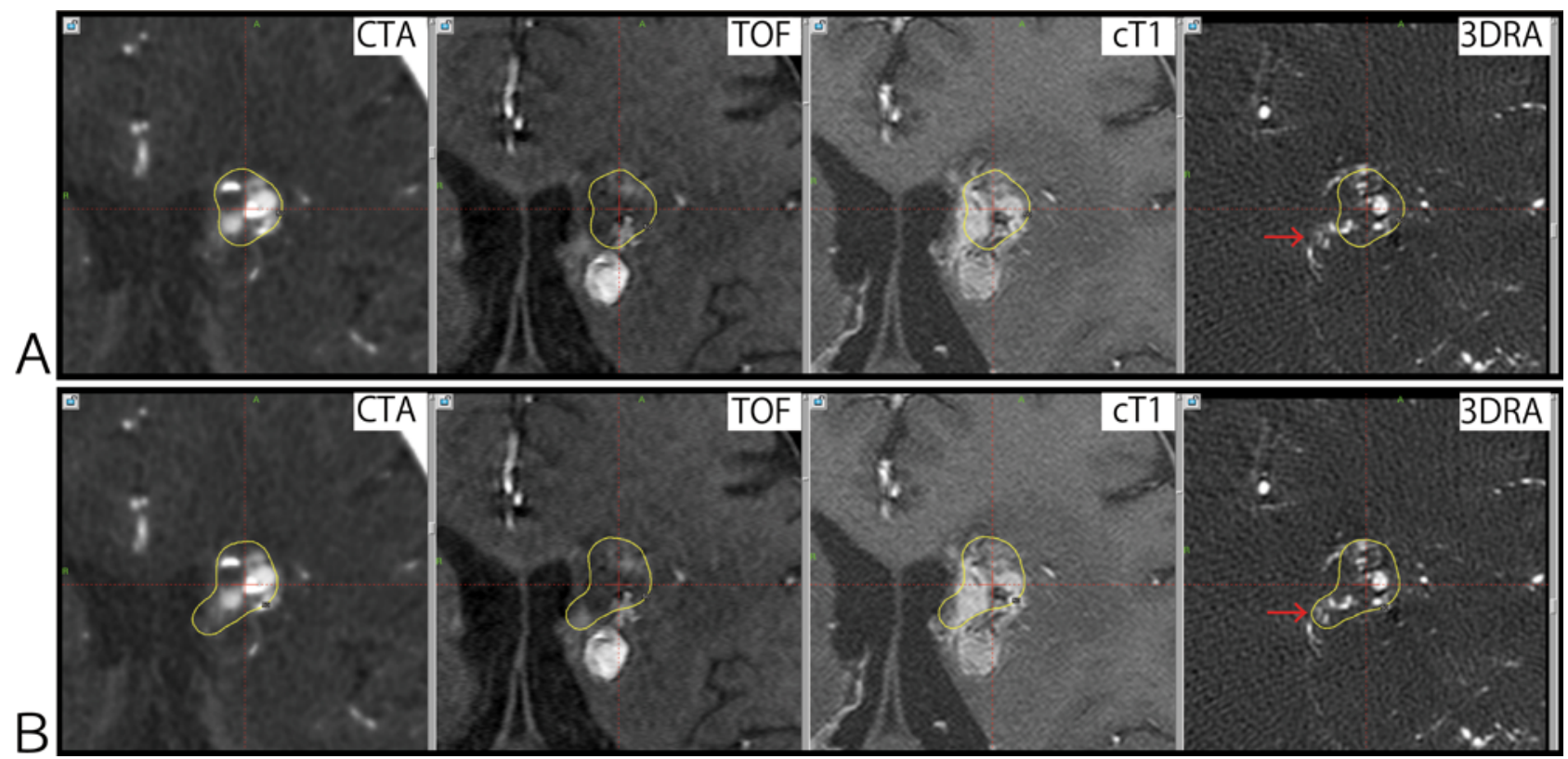

FIG. 2. Radiosurgical plans before (A) and after (B) the integration of 3DRA for a 62-year-old woman with a left basal ganglia AVM and a history of hemorrhage. The patient was referred to us 4 months after failed endovascular embolization using n-butyl2-cyanoacrylate, which resulted in an ischemic stroke that caused transient aphasia and right hemiparesis. A: The radiosurgical plan before the integration of 3DRA is based on the findings of CTA, because the nidus contour on MR images, including TOF and contrast-enhanced T1-weighted (cT1) images, is ambiguous due to artifacts from the embolized material and to the irregularity of the nidus caused by the previous hemorrhage and embolization. B: The radiosurgical plan created after the integration of 3DRA provides clearer pictures of the nidus, including the posteromedial part with very slender vessels (red arrow), which the other modalities failed to show. Yellow lines indicate the prescription isodose line.

performed herein have been performed previously. ${ }^{6,30}$ Our findings support the use of RA in GKS planning; however, further research aimed at determining whether the use of RA improves the radiosurgical outcomes, including the rates of nidus obliteration and complications, is needed.

The actual efficacy of RA seems to vary depending on the characteristics of the nidus. For patients with compact, usually sized niduses without any preceding events, the efficacy of RA might be limited because such niduses can also be visualized clearly on the current standard combination of DSA, CTA, and MR images. Indeed, the median (0.868 vs 0.890$)$ and maximum (0.945 vs 0.958$)$ UVRs were not that different from before to after the integration of RA, suggesting that MRI/CTA-based planning is already of relatively high quality. On the other hand, as shown in Figs. 2-5, RA integration would likely be the most effective in patients with ambiguous, small, and/or faint niduses that are difficult to visualize on the current standard combination of images. Notably, the minimum UVRs improved remarkably from before to after the integration of RA (0.498 to 0.730), suggesting that the integration of RA may prevent the undertreatment that is likely to occur in patients with ambiguous, small, and/ or faint niduses. Regarding prior events, all dosimetric variables improved regardless of prior radiotherapy and prior surgery/embolization; however, prior hemorrhage was significantly associated with larger improvement in the OVR, and ultimately in the CI. Hemorrhage can affect the signal intensity in MRI and the density in CT either adjacent to or inside the nidus, which might blur the nidus margin. Since RA can provide clear pictures of the angioarchitecture regardless of the intensity/density changes, this approach may contribute to better contouring. Further studies with more cases are desirable to confirm for which AVMs RA is the most beneficial. Since several previous studies have suggested that one of the major causes of radiosurgical treatment failure is targeting error, ${ }^{12,15,22,42,43}$ decreasing such undertreatment would be invaluable for better radiosurgical outcomes.

The OVRs in the present study were relatively low; however, this may be due to an overestimation of overtreatment. First, the definition of RTV was stricter than the actual target volume, as the RTV did not include drainers and feeders adjacent to the nidus, which many surgeons often include inside the target. Second, since the present study used the GammaPlan version 10.1.1 planning software, in which surgeons place one to dozens of ellipsoid "shots" with minimum diameters of approximately $4 \mathrm{~mm}$, perfect contouring was theoretically impossible for an irregularly shaped nidus with some sprawling parts $<4 \mathrm{~mm}$ in diameter. In such cases, undertreatment and overtreatment are related-that is, undertreatment can be decreased at the cost of an increase in overtreatment. Thus, surgeons should determine an optimal "balanced" plan in which target coverage is maximized and waste irradiation is minimized. In many cases, decreasing undertreatment should be prioritized to increase the obliteration rate; however, in some cases, suppressing overtreatment is important, especially when the nidus is at a critical location, such as the brainstem, thalamus, and basal ganglia. 

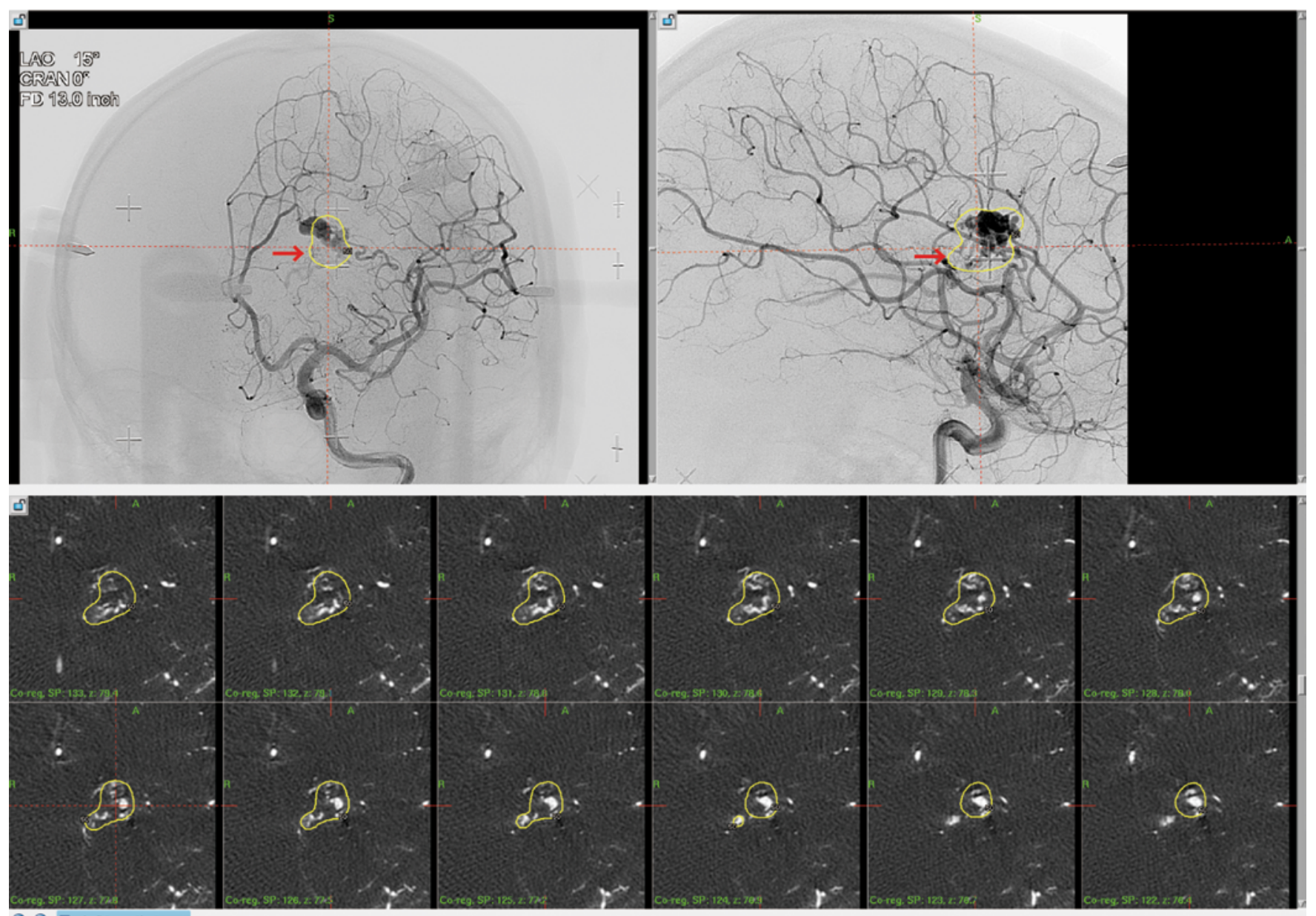

FIG. 3. The radiosurgical plan after the integration of 3DRA for the same patient as that in Fig. 1. DSA (upper) and serial axial 3DRA (lower) images are shown for comparison. Note that the parts of the nidus that could not be observed with DSA (red arrow) are visible in the 3DRA images.

Hence, the integration of RA adds value by allowing surgeons to ensure they are using the best strategy possible to clearly visualize the nidus.

Regarding radiation exposure, evidence suggests that the radiation exposure is lower for 3DRA than it is for a series of DSA or CTA images; the mean effective doses are roughly $0.9 \mathrm{mSv}$ for a single DSA acquisition, 2.1-3.4 $\mathrm{mSv}$ for a series of DSA acquisitions, 3-29 $\mathrm{mSv}$ for suspected-stroke CTA, and $0.2-1.3 \mathrm{mSv}$ for 3DRA..$^{5,8,31-33,35}$ As for HiRes-XperCT or cone-beam CTA, the speculated radiation exposure is roughly three times greater than that for 3DRA and two times greater than that for a single acquisition of biplane DSA. ${ }^{28}$ Our current angiography protocol entails a single acquisition of biplane DSA followed by RA, which mainly includes 3DRA, with the occasional use of HiRes-XperCT for cases with small, faint niduses; thus, it could be speculated that our protocol delivers an equivalent or a lower radiation dose than multiple magnified DSA acquisitions to obtain the appropriate images. ${ }^{30}$

This study has some limitations. First, we did not evaluate the "gradient," one of the other important dosimetric values indicating the quality of dose falloff outside the target, ${ }^{26,40}$ because the present study was conducted to ex- amine the effects of improved recognition of the angioarchitecture of AVMs owing to the integration of RA on target contouring. However, in reality, surgeons must consider the gradient, and thus this issue should be addressed in future studies. Second, we used a 50\% isodose line in this study to easily compare two rival plans. In some clinical cases, however, a higher $(60 \%-90 \%)$ or lower (down to $40 \%$ ) isodose line may be useful. In addition, owing to the sector system in newer-generation GK systems (ICON and PERFEXION; Elekta AB), where each of the 8 sectors of sources can move independently, surgeons can create a "composite shot," which is a single isocenter composed of different beam diameters. Such a composite shot allows an optimized dose distribution shape for individual shots, enabling more-tailored planning. Thus, future studies are needed to determine whether a combination of ICON or PERFEXION GKS and RA can provide more conformal radiosurgery. Third, intra- and interobserver variabilities should be taken into account when interpreting the results of the present study. Although we could not fully incorporate these variabilities, we did at least partially address them by using the standardized contouring protocol and allowing the physicians to use multimodal imaging., 

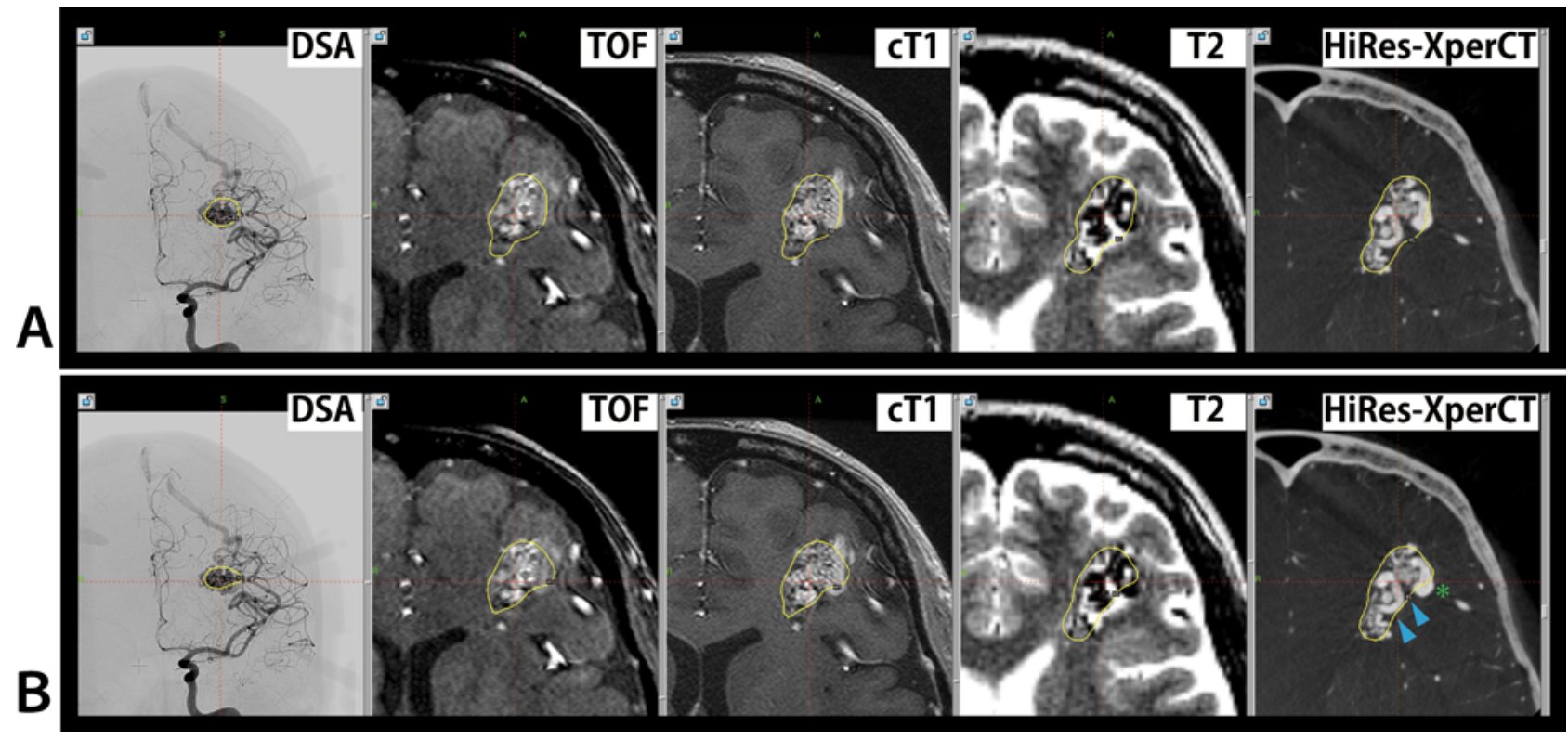

FIG. 4. The radiosurgical plans before $(\mathbf{A})$ and $\operatorname{after}(\mathbf{B})$ the integration of HiRes-XperCT for a 43-year-old woman with an unruptured left frontal AVM. The nidus is $13 \times 27 \times 12 \mathrm{~mm}$. In this patient, little difference is observed between the two plans because the nidus is so compact that it can be observed on all image types, including DSA, TOF, contrast-enhanced T1-weighted (cT1), and T2-weighted images. Nevertheless, the nidus can be observed more clearly on HiRes-XperCT images than on the other image types, enabling sharper demarcation of the lateral margin (blue arrowheads) and avoiding irradiation to an extranidal drainer (green asterisk).

Moreover, despite the potential intra- and interobserver variabilities, the integration of RA would have a certain fundamental benefit considering that RA can visualize some portions of niduses that are difficult to identify using other conventional imaging studies. Finally, considering that our sample size was not very large, further studies with more cases are required to validate our results.

\section{Conclusions}

The integration of RA in GKS planning permitted clear

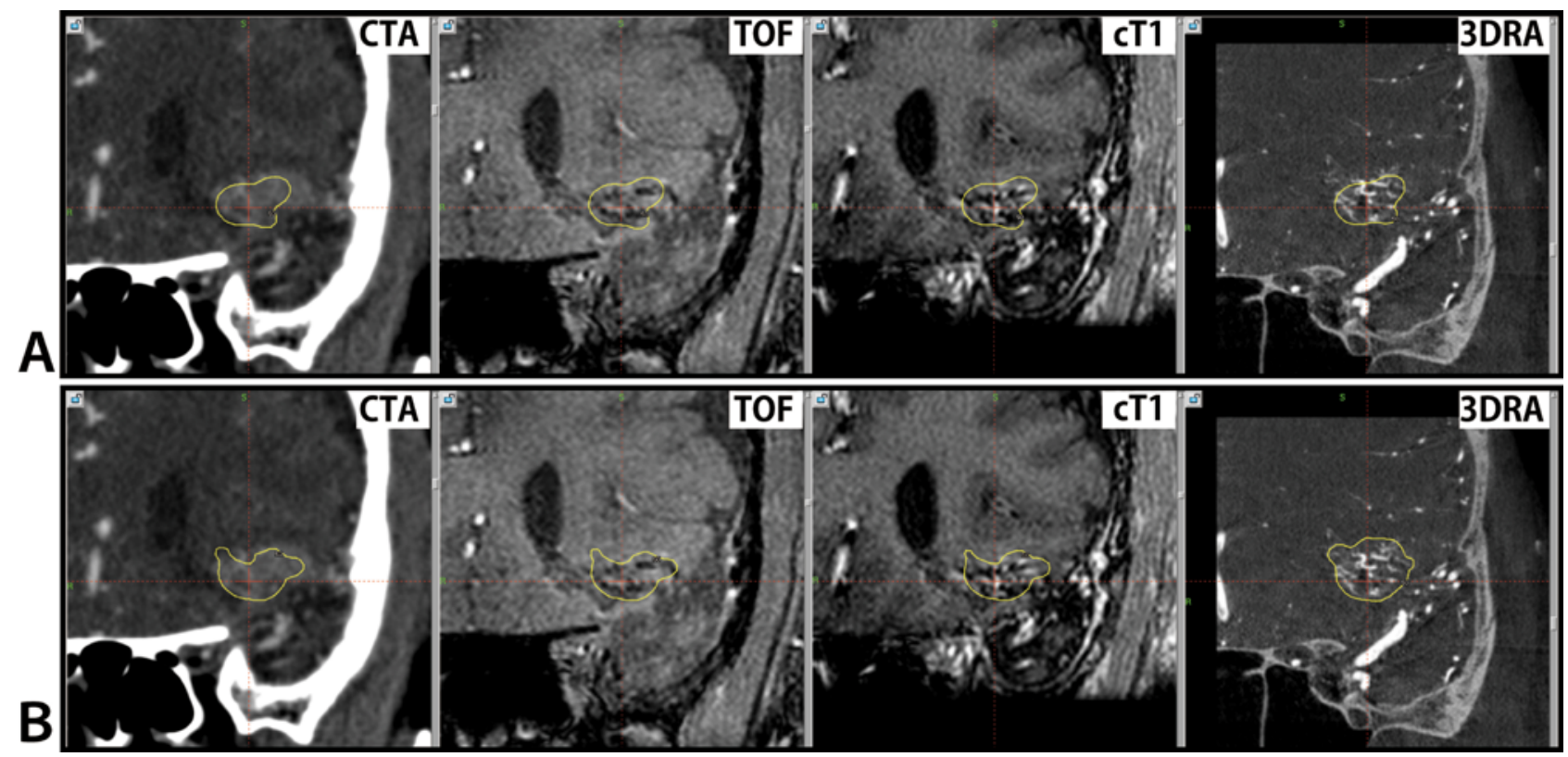

FIG. 5. The radiosurgical plans before $(\mathbf{A})$ and after $(\mathbf{B})$ the integration of 3DRA for a 53-year-old man with a ruptured left frontal AVM. The nidus is $17 \times 13 \times 13 \mathrm{~mm}$ and has a very diffuse appearance. A: Before the integration of 3DRA, the radiosurgical plan is created by meticulously comparing CTA, TOF, and contrast-enhanced T1-weighted (cT1) images, though significant portions of the nidus are undetected. B: After the integration of 3DRA, these portions of the nidus are clearly visualized. 
visualization of the nidus and reduced suboptimal coverage and waste irradiation, ultimately improving conformity of the radiosurgical plans. The efficacy of RA integration is maximal for ambiguous, small, and/or faint niduses that are difficult to visualize on MR or CTA images. Even though clearer visualization of the nidus is necessary for better dose planning, it is not always sufficient for perfectly contouring the nidus. As such, when creating their treatment plans, surgeons should pay careful attention to the nidus margin and adjacent structures. Further studies are required to examine whether the use of RA also improves the radiosurgical outcomes.

\section{Acknowledgments}

This study was supported by JSPS KAKENHI grant number JP17K16628 (to Hirotaka Hasegawa).

\section{References}

1. Ang CH, Low JR, Shen JY, Cai EZ, Hing EC, Chan YH, et al: A protocol to reduce interobserver variability in the computed tomography measurement of orbital floor fractures. Craniomaxillofac Trauma Reconstr 8:289-298, 2015

2. Armstrong J, McGibney C: The impact of three-dimensional radiation on the treatment of non-small cell lung cancer. Radiother Oncol 56:157-167, 2000

3. Armstrong J, Raben A, Zelefsky M, Burt M, Leibel S, Burman C, et al: Promising survival with three-dimensional conformal radiation therapy for non-small cell lung cancer. Radiother Oncol 44:17-22, 1997

4. Blatt DR, Friedman WA, Bova FJ: Modifications based on computed tomographic imaging in planning the radiosurgical treatment of arteriovenous malformations. Neurosurgery 33:588-596, 1993

5. Bridcut RR, Murphy E, Workman A, Flynn P, Winder RJ: Patient dose from 3D rotational neurovascular studies. Br J Radiol 80:362-366, 2007

6. Chen KK, Guo WY, Yang HC, Lin CJ, Wu CF, Gehrisch S, et al: Application of time-resolved 3D digital subtraction angiography to plan cerebral arteriovenous malformation radiosurgery. AJNR Am J Neuroradiol 38:740-746, 2017

7. Cohen-Inbar O, Starke RM, Kano H, Bowden G, Huang P, Rodriguez-Mercado R, et al: Stereotactic radiosurgery for cerebellar arteriovenous malformations: an international multicenter study. J Neurosurg 127:512-521, 2017

8. Cohnen M, Wittsack HJ, Assadi S, Muskalla K, Ringelstein A, Poll LW, et al: Radiation exposure of patients in comprehensive computed tomography of the head in acute stroke. AJNR Am J Neuroradiol 27:1741-1745, 2006

9. Colombo F, Cavedon C, Francescon P, Casentini L, Fornezza U, Castellan L, et al: Three-dimensional angiography for radiosurgical treatment planning for arteriovenous malformations. J Neurosurg 98:536-543, 2003

10. Conti A, Pontoriero A, Faragò G, Midili F, Siragusa C, Granata F, et al: Integration of three-dimensional rotational angiography in radiosurgical treatment planning of cerebral arteriovenous malformations. Int J Radiat Oncol Biol Phys 81:e29-e37, 2011

11. Dearnaley DP, Khoo VS, Norman AR, Meyer L, Nahum A, Tait D, et al: Comparison of radiation side-effects of conformal and conventional radiotherapy in prostate cancer: a randomised trial. Lancet 353:267-272, 1999

12. Ellis TL, Friedman WA, Bova FJ, Kubilis PS, Buatti JM: Analysis of treatment failure after radiosurgery for arteriovenous malformations. J Neurosurg 89:104-110, 1998

13. Fleetwood IG, Steinberg GK: Arteriovenous malformations. Lancet 359:863-873, 2002
14. Flickinger JC, Kondziolka D, Maitz AH, Lunsford LD: An analysis of the dose-response for arteriovenous malformation radiosurgery and other factors affecting obliteration. Radiother Oncol 63:347-354, 2002

15. Gallina P, Merienne L, Meder JF, Schlienger M, Lefkopoulos D, Merland JJ: Failure in radiosurgery treatment of cerebral arteriovenous malformations. Neurosurgery 42:996-1004, 1998

16. Giraud P, Helfre S, Lavole A, Rosenwald JC, Cosset JM: [Non-small-cell bronchial cancers: improvement of survival probability by conformal radiotherapy.] Cancer Radiother 6 (Suppl 1):125s-134s, 2002 (Fr)

17. Hanakita S, Koga T, Shin M, Igaki H, Saito N: The long-term outcomes of radiosurgery for arteriovenous malformations in pediatric and adolescent populations. J Neurosurg Pediatr 16:222-231, 2015

18. Kang J, Huang J, Gailloud P, Rigamonti D, Lim M, Bernard $\mathrm{V}$, et al: Planning evaluation of C-arm cone beam CT angiography for target delineation in stereotactic radiation surgery of brain arteriovenous malformations. Int J Radiat Oncol Biol Phys 90:430-437, 2014

19. Kano H, Kondziolka D, Flickinger JC, Yang HC, Flannery TJ, Awan NR, et al: Stereotactic radiosurgery for arteriovenous malformations, part 2: management of pediatric patients. J Neurosurg Pediatr 9:1-10, 2012

20. Kano H, Lunsford LD, Flickinger JC, Yang HC, Flannery TJ, Awan NR, et al: Stereotactic radiosurgery for arteriovenous malformations, part 1: management of Spetzler-Martin Grade I and II arteriovenous malformations. J Neurosurg 116:1120,2012

21. Kondziolka D, Lunsford LD, Kanal E, Talagala L: Stereotactic magnetic resonance angiography for targeting in arteriovenous malformation radiosurgery. Neurosurgery 35:585-591, 1994

22. Kwon Y, Jeon SR, Kim JH, Lee JK, Ra DS, Lee DJ, et al: Analysis of the causes of treatment failure in Gamma Knife radiosurgery for intracranial arteriovenous malformations. J Neurosurg 93 (Suppl 3):104-106, 2000

23. Lee WR, Hanks GE, Hanlon AL, Schultheiss TE, Hunt MA: Lateral rectal shielding reduces late rectal morbidity following high dose three-dimensional conformal radiation therapy for clinically localized prostate cancer: further evidence for a significant dose effect. Int J Radiat Oncol Biol Phys 35:251-257, 1996

24. Lunsford LD, Niranjan A, Kano H, Kondziolka D: The technical evolution of gamma knife radiosurgery for arteriovenous malformations. Prog Neurol Surg 27:22-34, 2013

25. Paddick I: A simple scoring ratio to index the conformity of radiosurgical treatment plans. Technical note. J Neurosurg 93 (Suppl 3):219-222, 2000

26. Paddick I, Lippitz B: A simple dose gradient measurement tool to complement the conformity index. J Neurosurg 105 (Suppl):194-201, 2006

27. Patel NV, Gounis MJ, Wakhloo AK, Noordhoek N, Blijd J, Babic D, et al: Contrast-enhanced angiographic cone-beam CT of cerebrovascular stents: experimental optimization and clinical application. AJNR Am J Neuroradiol 32:137-144, 2011

28. Rahal JP, Malek AM: Benefit of cone-beam computed tomography angiography in acute management of angiographically undetectable ruptured arteriovenous malformations. J Neurosurg 119:1015-1020, 2013

29. Régis J, Tamura M, Delsanti C, Roche PH, Pellet W, Thomassin JM: Hearing preservation in patients with unilateral vestibular schwannoma after gamma knife surgery. Prog Neurol Surg 21:142-151, 2008

30. Safain MG, Rahal JP, Raval A, Rivard MJ, Mignano JE, Wu JK, et al: Use of cone-beam computed tomography angiography in planning for gamma knife radiosurgery for 
arteriovenous malformations: a case series and early report Neurosurgery 74:682-696, 2014

31. Schueler BA, Kallmes DF, Cloft HJ: 3D cerebral angiography: radiation dose comparison with digital subtraction angiography. AJNR Am J Neuroradiol 26:1898-1901, 2005

32. Shkumat NA, Shroff MM, Muthusami P: Radiation dosimetry of 3D rotational neuroangiography and 2D-DSA in children. AJNR Am J Neuroradiol 39:727-733, 2018

33. Smith-Bindman R, Lipson J, Marcus R, Kim KP, Mahesh M, Gould R, et al: Radiation dose associated with common computed tomography examinations and the associated lifetime attributable risk of cancer. Arch Intern Med 169:20782086, 2009

34. Starke RM, Ding D, Kano H, Mathieu D, Huang PP, Feliciano $\mathrm{C}$, et al: International multicenter cohort study of pediatric brain arteriovenous malformations. Part 2: outcomes after stereotactic radiosurgery. J Neurosurg Pediatr 19:136-148, 2017

35. Tonkopi E, Al-Habsi AH, Shankar JJ: Radiation dose from $3 \mathrm{D}$ rotational vs. conventional $2 \mathrm{D}$ digital subtraction angiography in intracranial aneurysm coiling. Can J Neurol Sci 42:176-180, 2015

36. van der Bom IM, Gounis MJ, Ding L, Kuhn AL, Goff D, Puri AS, et al: Target delineation for radiosurgery of a small brain arteriovenous malformation using high-resolution contrastenhanced cone beam CT. J Neurointerv Surg 6:e34, 2014

37. van Rooij WJ, Sprengers ME, de Gast AN, Peluso JP, Sluzewski M: 3D rotational angiography: the new gold standard in the detection of additional intracranial aneurysms. AJNR Am J Neuroradiol 29:976-979, 2008

38. Veeravagu A, Hansasuta A, Jiang B, Karim AS, Gibbs IC, Chang SD: Volumetric analysis of intracranial arteriovenous malformations contoured for CyberKnife radiosurgery with 3-dimensional rotational angiography vs computed tomography/magnetic resonance imaging. Neurosurgery 73:262 270, 2013

39. Vinod SK, Min M, Jameson MG, Holloway LC: A review of interventions to reduce inter-observer variability in volume delineation in radiation oncology. J Med Imaging Radiat Oncol 60:393-406, 2016

40. Wagner TH, Bova FJ, Friedman WA, Buatti JM, Bouchet LG, Meeks SL: A simple and reliable index for scoring rival stereotactic radiosurgery plans. Int J Radiat Oncol Biol Phys 57:1141-1149, 2003

41. Weltens C, Menten J, Feron M, Bellon E, Demaerel P, Maes
$\mathrm{F}$, et al: Interobserver variations in gross tumor volume delineation of brain tumors on computed tomography and impact of magnetic resonance imaging. Radiother Oncol 60:49-59, 2001

42. Yamamoto M, Ide M, Jimbo M, Takakura K, Lindquist C, Steiner L: Neuroimaging studies of postobliteration nidus changes in cerebral arteriovenous malformations treated by gamma knife radiosurgery. Surg Neurol 45:110-122, 1996

43. Yamamoto M, Jimbo M, Hara M, Saito I, Mori K: Gamma knife radiosurgery for arteriovenous malformations: longterm follow-up results focusing on complications occurring more than 5 years after irradiation. Neurosurgery 38:906914, 1996

\section{Disclosures}

The authors report no conflict of interest concerning the materials or methods used in this study or the findings specified in this paper.

\section{Author Contributions}

Conception and design: Hasegawa. Acquisition of data: Hasegawa, Kawashima, Takahashi, Suzuki, Shinya, Ono, Shojima. Analysis and interpretation of data: Hasegawa. Drafting the article: Hasegawa. Critically revising the article: Hanakita. Reviewed submitted version of manuscript: Hanakita, Shin, Kawashima, Takahashi, Suzuki, Shinya, Ono, Shojima, Nakatomi, Saito. Approved the final version of the manuscript on behalf of all authors: Hasegawa. Statistical analysis: Hasegawa. Administrative/techni$\mathrm{cal} / \mathrm{material} \mathrm{support:} \mathrm{Kawashima,} \mathrm{Takahashi,} \mathrm{Suzuki,} \mathrm{Kin,} \mathrm{Ono,}$ Shojima. Study supervision: Shin, Nakatomi, Saito.

\section{Supplemental Information}

\section{Previous Presentations}

Portions of this work were presented in an oral session at the 19th Leksell Gamma Knife Society Meeting, Dubai, March 4-8, 2018.

\section{Correspondence}

Hirotaka Hasegawa: University of Tokyo Hospital, Tokyo, Japan. hirohasegawa-tky@umin.ac.jp. 